\title{
Concentration, Temperature and Molecular Weight Dependent on Optical Properties of Poly (Vinyl Pyrrolidone) in Chloroform Solutions
}

\author{
Marwa. S. Alhosh, Zainab O. Ettarhouni*, Laila M. Abusen and Abdulhakim A. Jangher \\ Chemistry Department, Faculty of Science, University of Tripoli, \\ Tripoli City 34517, Libya
}

\begin{abstract}
We report on the concentration, temperature and molecular weight dependent of some optical properties of poly(vinyl pyrrolidone), PVP dissolves in chloroform for different molecular weights $(25 \mathrm{k}, 44 \mathrm{k}$ and $700 \mathrm{k})$ at different concentrations $(0.1-10 \mathrm{w} / \mathrm{v} \%)$ with temperature range $\left(25-45^{\circ} \mathrm{C}\right)$. Refractive index has been measured to determine the optical parameters including, reflectance, coefficient of fineness and critical angle. The results show that refractive index, reflectance and coefficient of fineness values increased with increasing concentration and molecular weight, whereas the temperature had a different influence. However, theses parameters were decreased with increasing the temperature. On the other hand, critical angle values the results presented that the refractive index, reflectance, and coefficient of fineness of the polymer decreases with increasing the temperature while the critical angle has the revers behavior.
\end{abstract}

Keywords: Poly(vinyl pyrrolidone), chloroform, polymer solutions, optical properties, refractive index, reflectance, coefficient of finesse, critical angle.

\section{INTRODUCTION}

Polymers are currently attracting considerable research attention because of their broad industrial applications. Basic polymer features such as easy production, low initial cost, durability and exceptional processability necessitate its use in several scientific applications ${ }^{[1,2]}$. Polymers in many instances can also tolerate easier shaping and better processing of the composite materials. Poly(vinyl pyrrolidone), PVP is one of the attractive polymers due to the strong affinity of the pyridine group and its ability to undergo the hydrogen bonding with polar species ${ }^{[3,4]}$. PVP does possess a unique combination of properties, such as environmental stability, with inert physicochemical properties over a broad range of $\mathrm{pH}$ values ${ }^{[5]}$, good solubility in both water and a range of organic solvents, a remarkable capacity to interact with a wide variety of organic and inorganic compounds, good biocompatibility, compatibility and resistance, and non-toxicity to living tissues. For these reasons PVP has been widely used in the biomaterials and coatings, pharmacy, cosmetics and industrial production, blood plasma substitute, which closely affect human health ${ }^{[6]}$. PVP has also been widely used as a medical additive or polymeric modifier. In fact, PVP is one of the most frequently investigated classes of materials for usage in medicine and in other applications interfacing with biological systems ${ }^{[7-10]}$. Another advantage is that PVP large scale screen printing of PVP films at low cost is feasible and it is also used in detergent formulations where its role is to prevent re-deposition of soil on fibers. PVP can be thermally cross-linked, thus ensuring a good thermal stability and mechanical strength and also because of the stability of PVP in water ${ }^{[11,12]}$. It should be noted that the formation of the transition metal particles inside the polymer system is also of interest for some potential applications, including the storage of optical data, shielding of the electromagnetic radiations, as flexible elements for resistive heating, laser systems, optical lenses and integrated waveguides, easy processing, and moderate electric conductivity for batteries and displays ${ }^{[13-15]}$. The interactions between water-soluble polymers and metal ions have a significant influence on the polymer physical properties ${ }^{[16,17]}$. PVP thermally decompose before reaching its molten state. This terminates the application of this polymer. PVP is chosen as a matrix for the composites because of the two important characteristics ${ }^{[18]}$. PVP has good film-forming and adhesive behavior on many solid substrates and its formed films exhibits good optical excellence (high transmission in visible range), and mechanical strength (easy dispensation) required for application ${ }^{[7]}$. The amorphous structure of PVP also provides a low scattering loss, which makes it as an ideal polymer for composite materials for different applications. PVP is easily soluble in water, so it is preferred to avoid phase separation in the reactions ${ }^{[8,9,19]}$. The PVP polymer film has a potential capacity to store the charges responding to the dopant dependent electrical and optical properties. It has a strong tendency for complex formation with a variety of molecules ${ }^{[10]}$. In literature, alkali ions containing polymers are reported to be more promising possessing potential applications ${ }^{[20,21]}$.

\section{Materials}

\section{EXPERIMENTAL METHODS}

The three molecular weights 25k, 44k and 700k of PVP were obtained from BDH Limited England, and were dried under vacuum at $60^{\circ} \mathrm{C}$ before use. Chloroform was of Riedel, Dehaen and Hannover product, monobromo naphthalene. 


\section{Preparation of solutions}

PVP-25k, 44k and 700k solutions with different weight per volume percentage of $25 \mathrm{k}$ and $44 \mathrm{k},(0.1-10 \mathrm{w} / \mathrm{v} \%)$ and $700 \mathrm{k}$ with range $(0.1-5.0 \mathrm{w} / \mathrm{v} \%) \mathrm{g} / \mathrm{ml}$ have been prepared by dissolving the propagate weights of PVP-25k, 44k and 700k powder in a fixed volume $(100 \mathrm{ml})$ of chloroform by using magnetic stirrer in mixing process for $15 \mathrm{~min}$ to get homogeneous solutions.

\section{Refractive index measurements}

The refractive index measurements were carried out using the refractometer type Care Zeiss/Germany. The temperatures of measured solutions were controlled within $0.5^{\circ} \mathrm{C}$. The refractometer was checked by standard material monobromo naphthalene.

\section{RESULTS AND DISCUSSION}

Refractive index has been measured for ten different concentrations (0.1-10\%) of PVP in chloroform solution of the molecular weights, $25 \mathrm{k}$ and $44 \mathrm{k}$ and for seven concentrations (0.1-5.0\%) of PVP for the molecular weight 700k in chloroform solution. All solutions were measured at five different temperatures $\left(25-45^{\circ} \mathrm{C}\right)$ at $5^{\circ} \mathrm{C}$ intervals. The optical constant such as reflectance, coefficient of finesse and critical angle were calculated also for all above-mentioned concentrations at the same temperatures. The results of the analysis of obtained data were discussed by looking at the general behavior of the considered optical properties as a function of concentration, temperature and molecular weight.

\section{Refractive index $\left(n_{D}\right)$}

Refractive index is one of the most important optical properties of a medium. It plays vital role in many areas of material science with special reference to thin film technology and fiber optics. Similarly, measurement of refractive index is widely used in analytical chemistry to determine the concentration of solutions.

Refractive index is one of the most important optical properties of a medium. It plays vital role in many areas of material science with special reference to thin film technology and fiber optics. Similarly, measurement of refractive index is widely used in analytical chemistry to determine the concentration of solutions.

Refractive index is one of the most important optical properties of a medium. It plays vital role in many areas of material science with special reference to thin film technology and fiber optics. Similarly, measurement of refractive index is widely used in analytical chemistry to determine the concentration of solutions. The refractive index is defined as the ratio between the speed of light (c) in a vacuum to the speed (v) of any center of a certain length and a certain wavelength, gives the following equation [22, 23]:

$$
n_{D}=\frac{v}{c}
$$

The refractive indices of all PVP solutions were measured at the same temperatures range mentioned above, and the values of at least four readings being averaged, for the three different molecular weights $25 \mathrm{k}, 44 \mathrm{k}$ and $700 \mathrm{k}$ respectively.

\subsection{Concentration effect}

To study the effect of the concentration on refractive index $\left(\mathrm{n}_{\mathrm{D}}\right)$ for each system of PVP in chloroform solutions was plotted against concentration for three systems and at selected temperature $35^{\circ} \mathrm{C}$, as an example. The refractive index linearly increasing with the increasing of PVP concentration as shown in Figure (1), this behavior attribute to increase of the density with increase the concentration ${ }^{[24,25]}$.

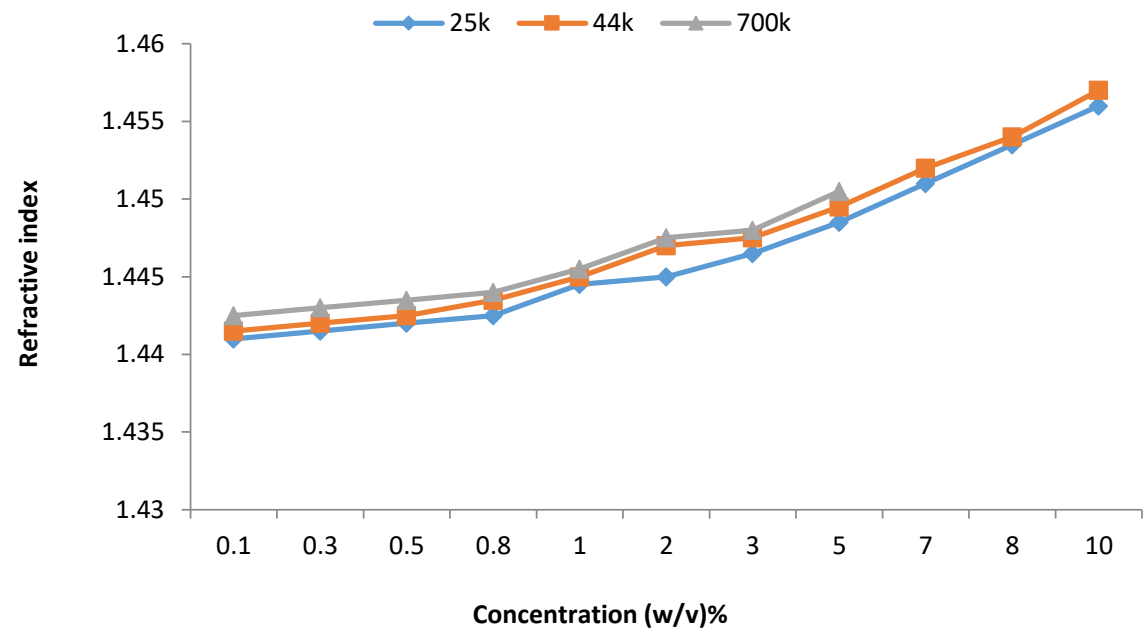

Figure (1): Concentration dependence of Refractive index of PVP in chloroform solutions at $35^{\circ} \mathrm{C}$ for three different molecular weights.

\subsection{Temperature effect}

The variations of refractive indices with temperature are evaluated at 3.0\% PVP. Figure (2) shows the refractive index as a function of temperature. It is clear that the refractive index of the polymer decreases with increasing temperature, and the 
results showed linear relationship between refractive index and temperature of the polymer within the ranges of temperature, this behavior is due to the temperature effect to activate of the kinetic atoms of the polymer, and this may be due to the fact that increasing temperature decreases the density of the solution ${ }^{[26]}$.

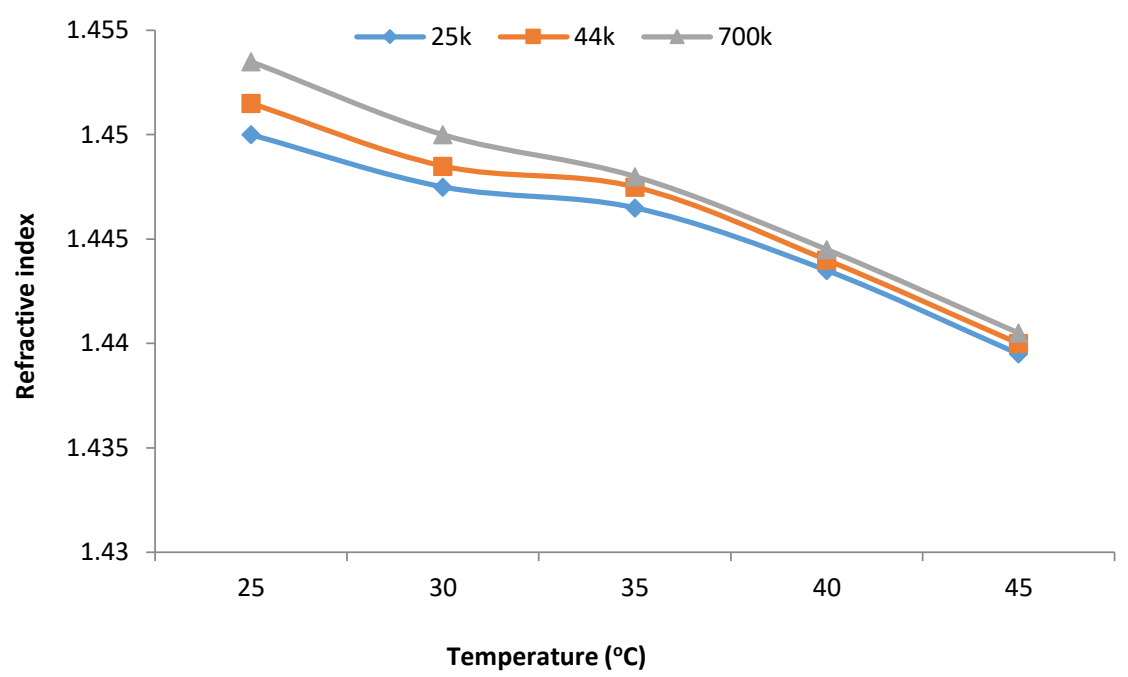

Figure (2): Temperature dependence of Refractive index of 3.0\% PVP in chloroform solutions for three different molecular weights of.

\subsection{Molecular weight effect}

The refractive index dependence on the molecular weight of PVP in chloroform, for selected three concentrations $(0.3$, 2.0 and $5.0 \%$ ) and temperature $35^{\circ} \mathrm{C}$ as an example, is demonstrated in Figure (3). It can be seen that the increasing of molecular weight rather rapid increase in the refractive index of these solutions. This general increase of refractive index with molecular weight maybe due to the increase of polar side groups in the molecular chain of the polymer ${ }^{[27]}$.

\section{Reflectance $(R)$}

When light radiation passes from one medium into another having a different index of refraction, some of the light is scattered at the interface between the two media even if both are transparent . The reflectance can be represented depending on the value of refractive index by using equation (2) ${ }^{[24]}$, which the reflectivity of PVP solutions was studied for concentrations and temperature of the three different molecular weights $(25 \mathrm{k}, 44 \mathrm{k}$ and $700 \mathrm{k})$ respectively:

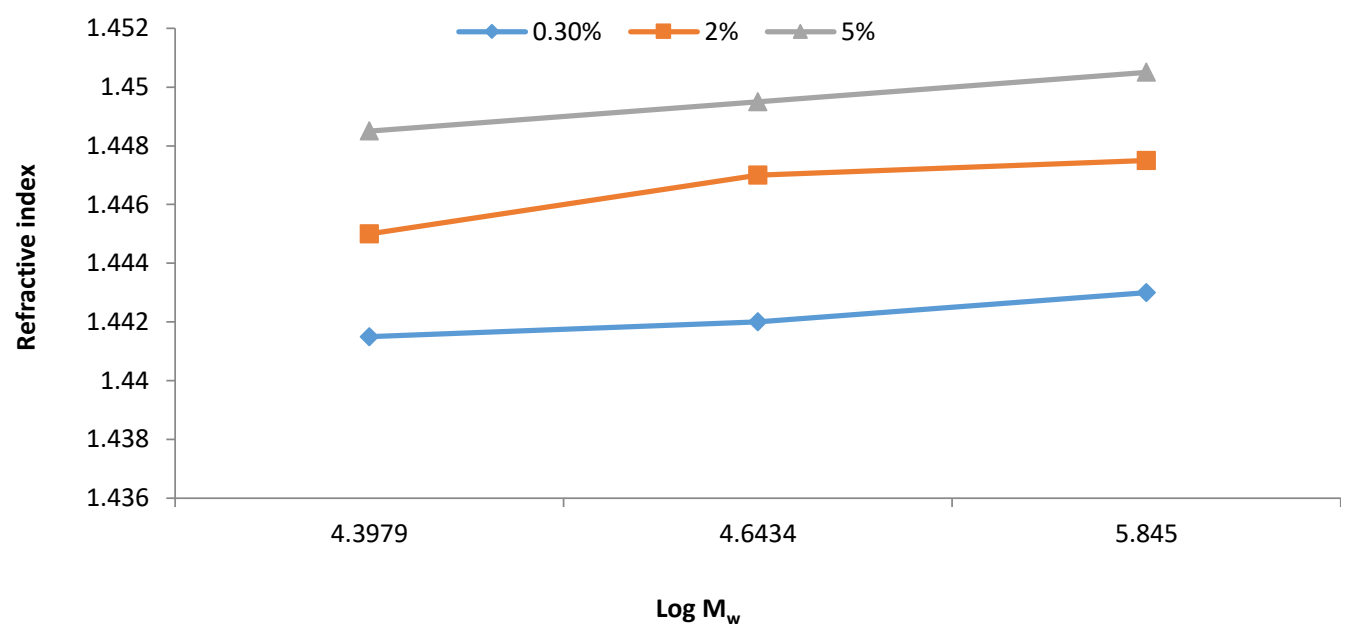

Figure (3): Molecular weight dependence of Refractive index of PVP in chloroform solutions at $35^{\circ} \mathrm{C}$ for three different concentrations.

$$
\mathbf{R}=\left[\frac{\mathbf{n}-\mathbf{1}}{\mathbf{n}+\mathbf{1}}\right]^{2}
$$




\subsection{Concentration Effect}

Reflectance of all PVP samples was computed by using equation (2). The results of reflectance were plotted in the Figure (4). It shows to have the same behavior of their refractive index, because reflectance equation has only one variable parameter. It's a clear from Figure below the values of reflectance are increases with increasing concentration, the reason for this is due to the increased number of polymer molecules in solution and therefore increase the density of the solution as the reflectiveness is entirely dependent on the density ${ }^{[24]}$.

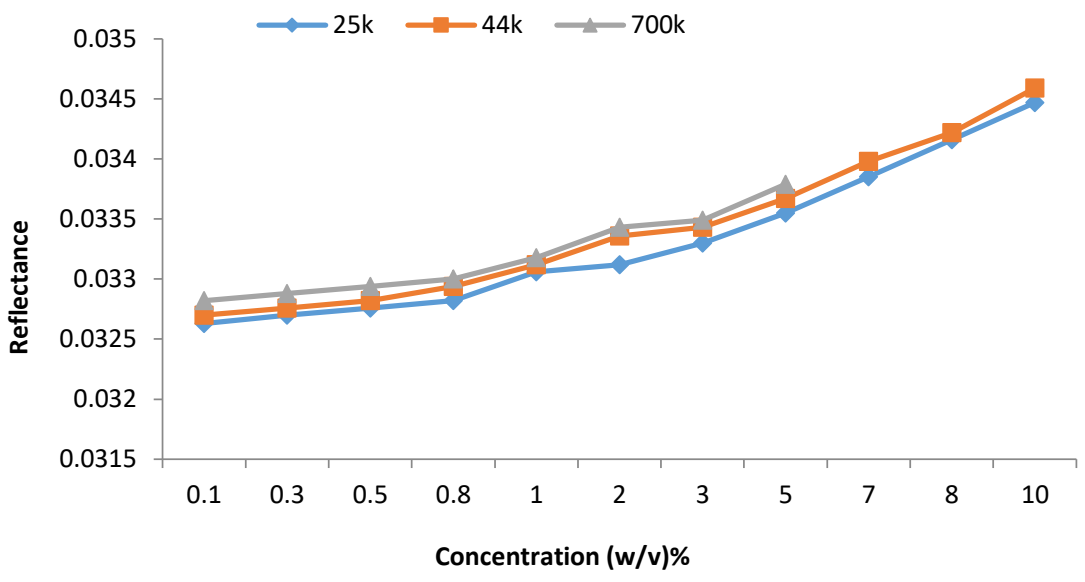

Figure (4): Concentration dependence of Reflectance of PVP in chloroform solutions at $35^{\circ} \mathrm{C}$ for three different molecular weights.

\subsection{Temperature effect}

Figure 5 illustrates the dependence of reflectivity on temperatures investigated with selected 3.0\% PVP of three systems. It indicates that at the fixed concentration, the refractive index decrease linearly with the temperatures $\left(25-45^{\circ} \mathrm{C}\right)$, and this is because that the increasing of the temperature leads to decrease in the particles of solution in the volume unit and decrease the density of the solution ${ }^{[26,28]}$.

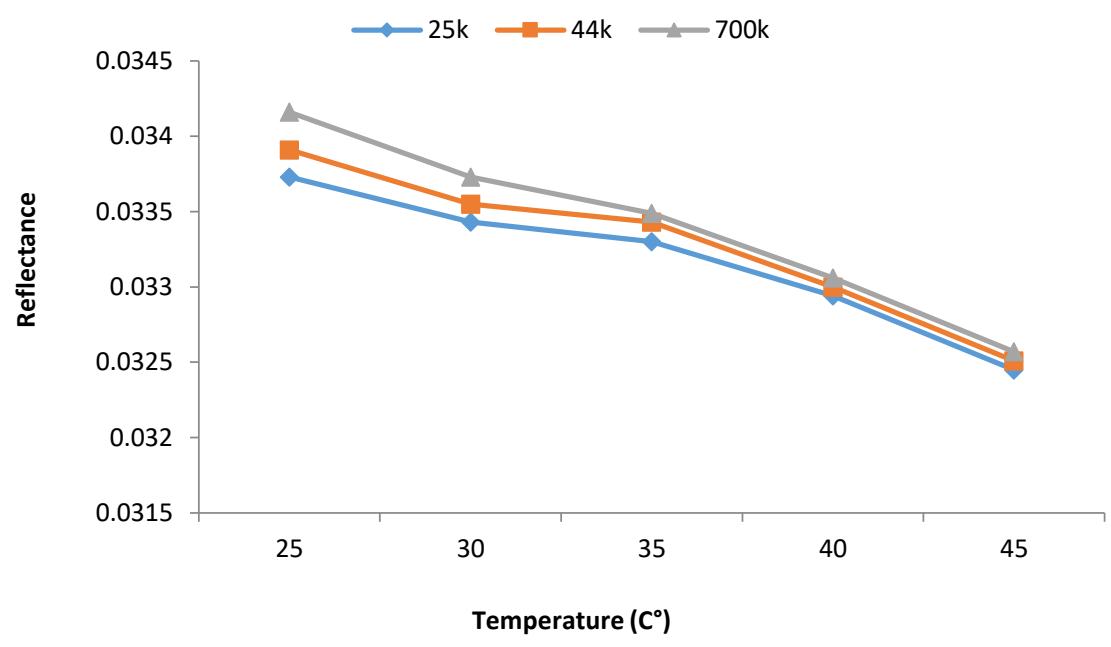

Figure (5): Temperature dependence of Reflectance of 3\% PVP in chloroform solutions for three different molecular weights.

\subsection{Molecular weight effect}

The effect of molecular weight on the reflectivity for each system was plotted against molecular weight for selected three concentrations $\left(0.3,1.0\right.$ and 5.0\%) and selected temperature $35^{\circ} \mathrm{C}$ as an example, this is demonstrated in Figure (6). It can be seen from this figure that the increasing of the molecular weight shows rather rapid increase in the reflectivity solution with molecular weight. This general increase of reflectivity with molecular weight maybe due to the increase of polar side groups in the molecular chain of this polymer ${ }^{[29]}$. 


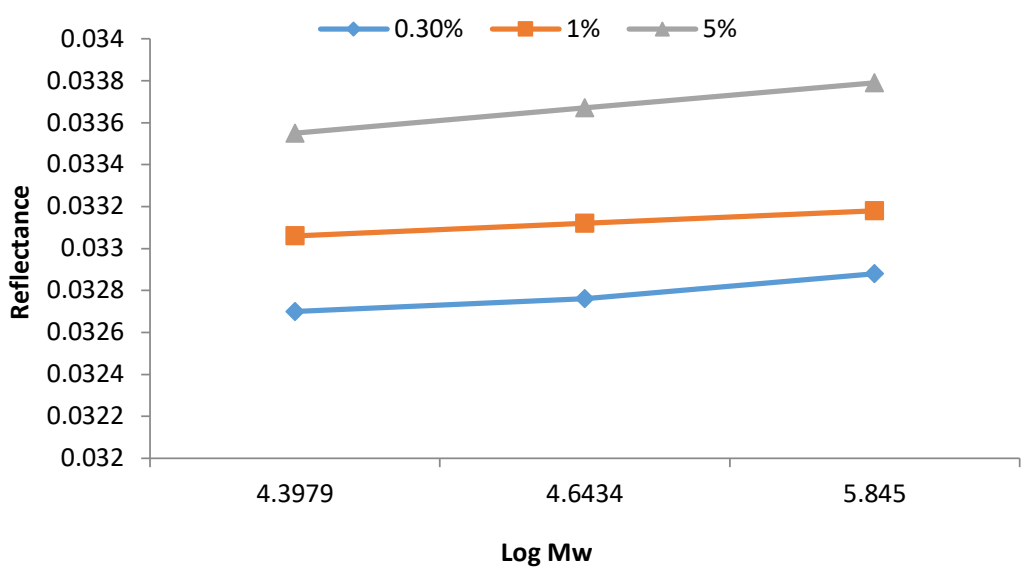

Figure (6): Molecular weight dependence of Reflectance of PVP in chloroform solutions at $35^{\circ} \mathrm{C}$ for three different molecular weights.

\section{COEFFICIENT OF FINESSE (F)}

The finesse coefficient is define as a measure of the severity of interference fringes and measured for the prepared samples by using the equation (3) ${ }^{[30]}$, and the coefficients of finesse values were obtained for all studied systems:

$$
F=\frac{4 R}{(1-R)^{2}}
$$

\subsection{Concentration Effect}

The curves between the finesse coefficient against concentration for three systems and at selected $35^{\circ} \mathrm{C}$ as an example, have been plotted in Figure (7), this Figure shows that the coefficient of finesse of all PVP samples have the same behavior to their reflectance, because this coefficient depends on reflectance, from another aspect the influences of addition lead to increase the values of finesse coefficient, this is because the increasing of reflected light as a result of increasing the density and this results agreement with reference ${ }^{[30]}$.

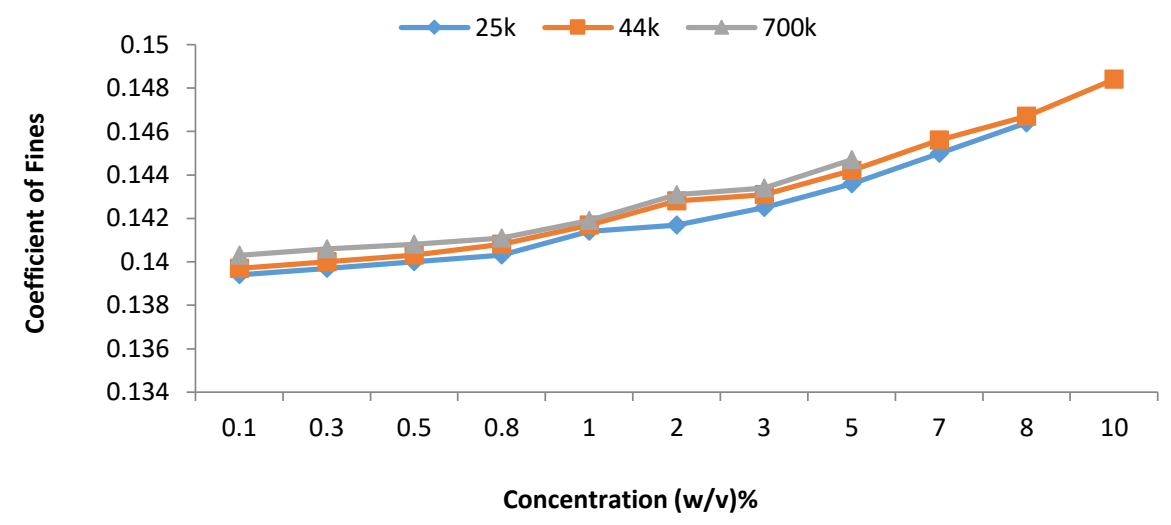

Figure (7): Concentration dependence of Coefficient of fine of PVP in chloroform solutions at $35^{\circ} \mathrm{C}$ for three different molecular weights.

\subsection{Temperature effect}

The coefficient of fine was also plotted against the temperature of 3\% PVP in chloroform solutions as an example, for three different molecular weights, which shown in Figure (8). It is obvious that the decrease of coefficient of fine with increasing the temperature is because the increase in temperature leads to a decrease in the particles of solution in the volume unit and decrease the density of the solution with increasing temperature ${ }^{[31]}$. 


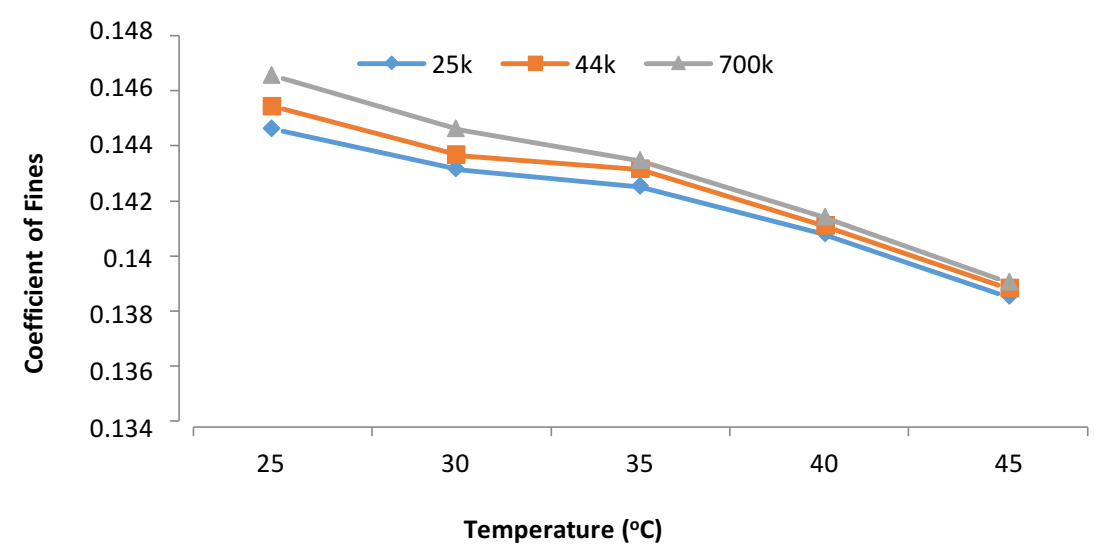

Figure (8): Temperature dependence of Coefficient of fines of 3\% PVP in chloroform solutions for three different molecular weights.

\subsection{Molecular weight Effect}

The coefficient of fines dependence on the molecular weight of the PVP in chloroform solution, for three selected concentrations $(0.3,1.0$ and $5.0 \%)$ and selected temperature $35^{\circ} \mathrm{C}$, as an example, which shown in Figure (9). It has been seen that that increasing molecular weight shows rather rapid increase in the coefficient of fines of solution. This general increase of coefficient of Fines with molecular weight maybe due to the increase of polar side groups in the molecular chain of this polymer.

\section{Critical Angle $\left(\Theta_{c}\right)$}

The critical angle is defined as the angle of incidence for which the angle of refraction is $90^{\circ}$ and then reflected light fully reflection or internal reflection. The critical angle is calculated using equation (4) ${ }^{[24,25]}$, for all the studied systems:

$$
\theta_{c}=\operatorname{Sin}^{-1}(1 / n)
$$

Where; $n$ is the refractive index of the solution.

\subsection{Concentration Effect}

The critical angle values decrease linearly with the increase of the concentration for three different molecular weights and at a selected $35^{\circ} \mathrm{C}$ as an example, as shown in Figure (10), because it is inversely proportional of their refractive index, or it is clear that as concentration increase, the density also increase and causes critical angle to decrease and this is agreement with [25].

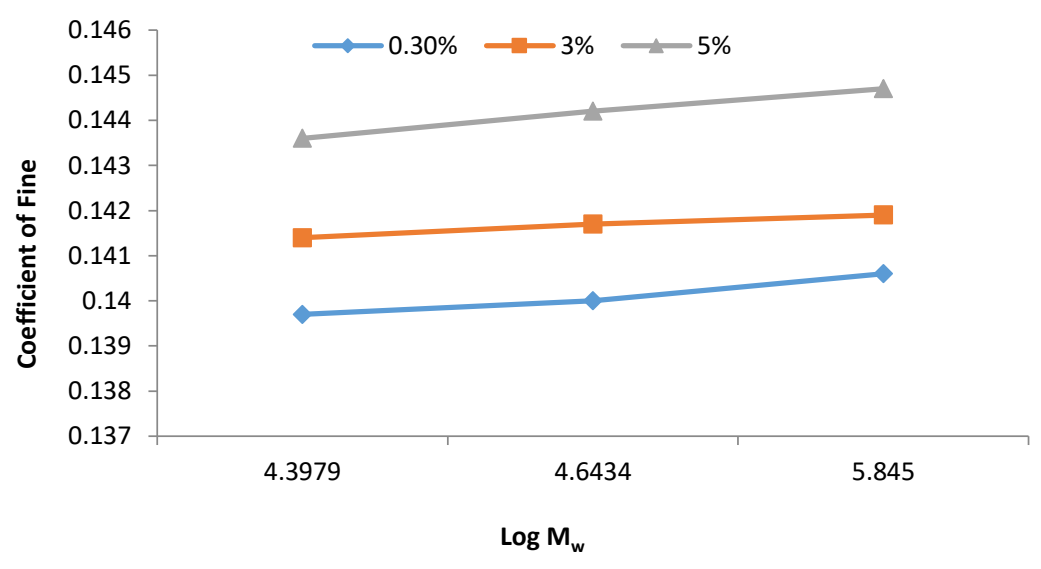

Figure (9): Molecular weight dependence of Coefficient of fines of PVP in chloroform solutions at $35^{\circ} \mathrm{C}$ for three different molecular weights. 


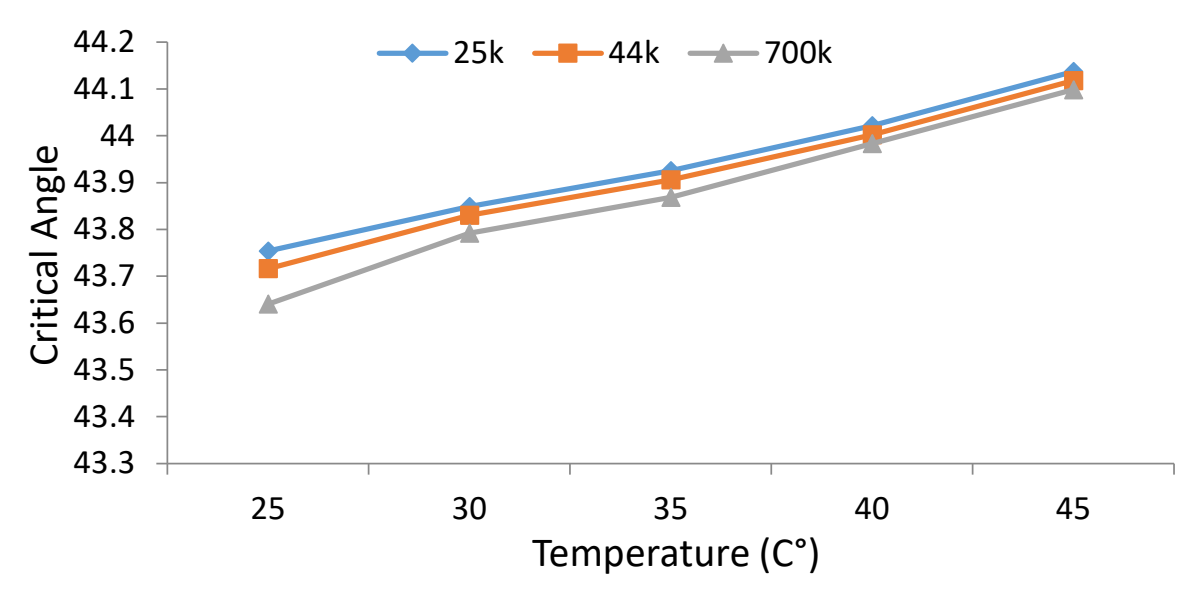

Figure (10): Concentration dependence of Critical angle at $35^{\circ} \mathrm{C}$ for three different molecular weights of PVP in chloroform solutions.

\subsection{Temperature Effect}

The critical angle were also plotted against temperature of PVP in chloroform solutions for three selected concentrations $(0.3,3.0$ and $5 \%)$ as an example for three different molecular weights, where it is found in Figures (11), which is shown the increase of the critical angle with the increase of the temperature due to the inverse relation between temperature and refractive index ${ }^{[32]}$.

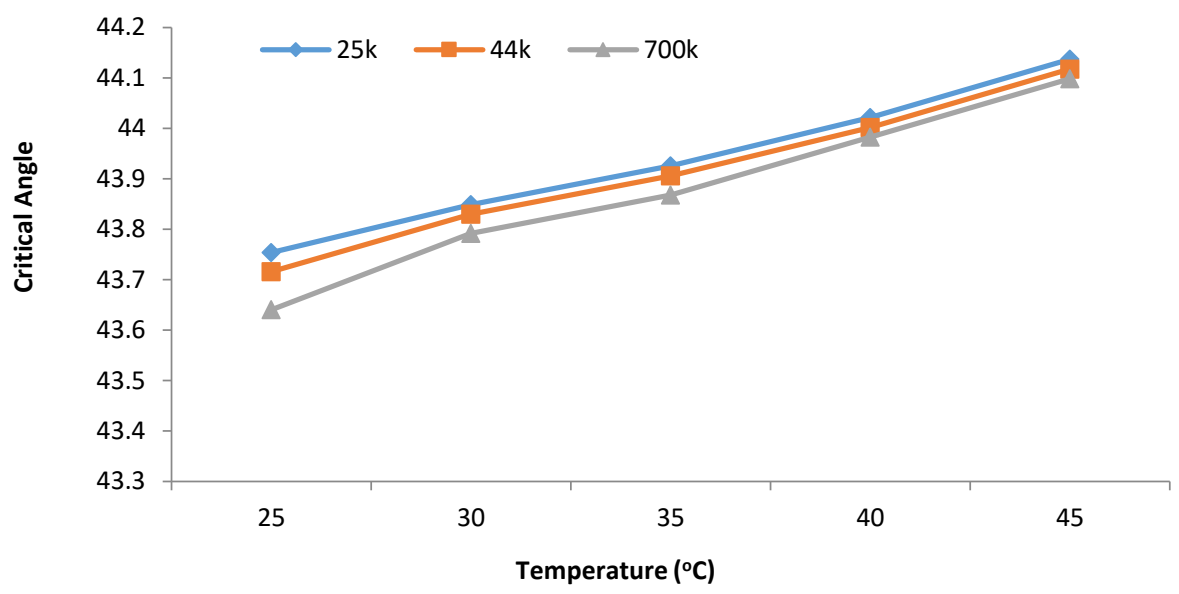

Figure (11): Temperature dependence of Critical angle of 3\% PVP in chloroform solutions for three different molecular weights.

\subsection{Molecular weight Effect}

The critical angle dependence on the molecular weight of the PVP in chloroform solutions, for three selected concentrations $(0.3,1.0$ and $5.0 \%)$ at selected temperature $35^{\circ} \mathrm{C}$ as an example, is demonstrated in Figure (12). It can be seen that increasing the molecular weight shows rather rapid decrease in the critical angle of solution. 


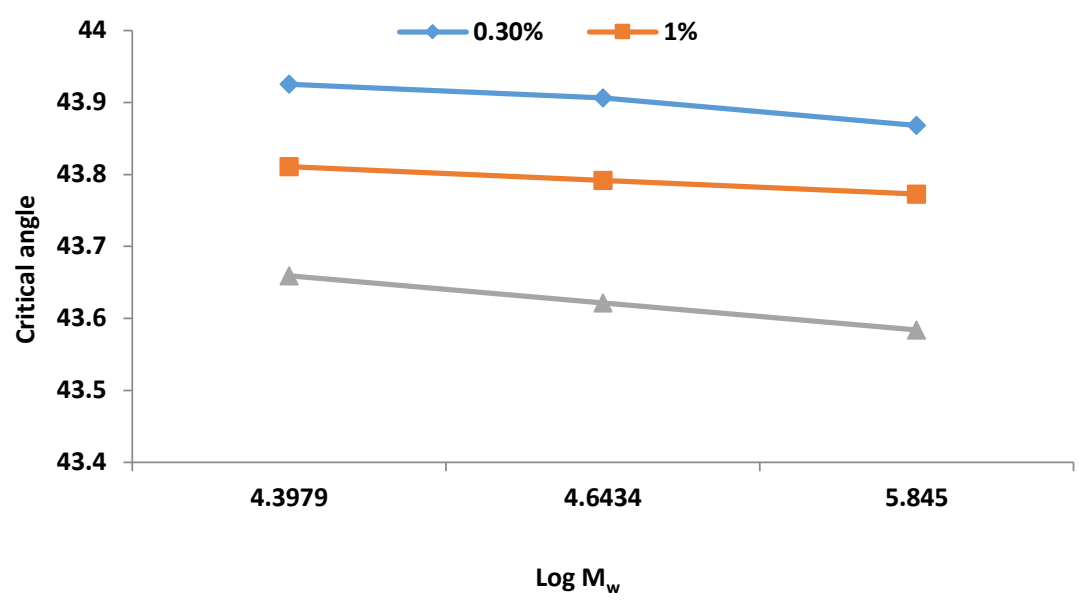

Figure (12): Molecular weight dependence of Critical angle of PVP in chloroform solutions at $35^{\circ} \mathrm{C}$ for three different molecular weights.

\section{CONCLUSION}

The optical properties of PVP in chloroform solutions for three molecular weights $(25 \mathrm{k}, 44 \mathrm{k}$ and $700 \mathrm{k})$ with different solutions concentration and with temperature range from $\left(25-45^{\circ} \mathrm{C}\right)$ have been investigated. These polymer solutions show a continuous change in their optical properties as a result of concentration increasing of PVP which led to development of these properties. Also the increasing concentration of PVP leads to increase in the refractive index and the reflectivity of the solution, thus it can be used to protect the human skin from the sun's rays and for the production of sunglasses so that the human eye can be protected from the risk of these rays.

\section{Credit authorship contribution statement}

Marwa. S. Alhosh: conceptualization, methodology, and writing - original draft, Zainab O. Ettarhouni: methodology. Laila M. Abusen: methodology and writing - review \& editing. Abdulhakim A. Jangher: conceptualization, methodology, writing - original draft and writing - review \& editing.

\section{ACKNOWLEDGEMENTS}

The authors would like to acknowledge the assistance offered using the refractometer type Care Zeiss, Department of Chemistry, Faculty of Science, University of Tripoli, Libya.

\section{REFERENCES}

[1] P. Ajayan, L. Schadler and P. Braun; "Nanocomposite science and technology"; Weilheim: WILEY-VCH Verlag GmbH \& Co. KGaA; (2006).

[2] J. Jordan, K. Jacob, R. Tannenbaum, M. Sharaf and I. Jasiuk; "Experimental trends in polymer nanocomposites-a review"; Mater Sci. Eng. A.; vol. 393, pp: 1-11(2005).

[3] N. Kumar; "Enhanced photoluminescence of $\mathrm{Mn}^{2+} \& \mathrm{~Tb}^{3+}$ ions doped PEO and PVP blended polymer films"; Proc. Indian Nat. Sci. Acad., vol. 80, pp: 345-354 (2014).

[4] A. Chandra, R. Agrawal and Y. Mahipal; "Ion transport property studies on PEO/PVP blended solid polymer electrolyte membranes"; J. Phys. D: Appl. Phys. vol. 42, pp:1-4 (2009)

[5] A. Kedia and P. Kumar; "Precursor-Driven Nucleation and Growth Kinetics of Gold Nanostars"; J. Phys. Chem. C; vol. 116, No. 2, pp: 1679-1686 (2012).

[6] M. Loría-Bastarrachea, W. Herrera-Kao, J. Cauich-Rodríguez, J. Cervantes-Uc, H. Vázquez-Torres and A. Ávila-Ortega, "A TG/FTIR study on the thermal degradation of poly(vinyl pyrrolidone)"; J. Therm. Anal. Calorim., vol. 104, pp: 737-742 (2011).

[7] W. Teo and S. Ramakrishna; "A review on electrospinning design and nanofibre assemblies; Nanotechnology, vol.17, pp: R89-R106 (2006).

[8] L. Wang, L. Zhang and M. Tian; "Improved poly(vinyl pyrrolidone),PVP/graphite nanocomposites by solution compounding and spray drying"; Polym. Adv. Technol., vol. 23, pp: 652-659 (2012).

[9] S. Agarwal, A. Greiner and J. Wendorff; "Functional materials by electrospinning of polymers"; Prog. Polym. Sci., vol. 38, pp: 963-991 (2013).

[10] N. Bhardwaj and S. Kundu; "Electrospinning: A fascinating fiber fabrication technique"; Biotechnol. Adv., vol. 28, pp: 325-347 (2010).

[11] S. Selvam and S. Mahalingam; "Functionalization of cotton fabric with PVP/ZnO nanoparticles for improved reactive dye ability and antibacterial activity Carbohydras"; Polym. , vol. 87, pp: 1419-1424 (2012).

[12] M. Ravi, Y. Pavani, K. Kumar, S. Bhavani, A. Sharma and V. Rao; "Studies on electrical and dielectric properties of PVP: KBrO ${ }_{4}$ complexed polymer electrolyte films"; Mater. Chem. Phys., vol.130, pp: 442 - 448 (2011).

[13] M. Ambalagi, S. Nagaraja, V. Manjula, S. Hogade, H. Inamdar, M. Ambikaprasad and B. Sannakki "Dielectric properties of poly(vinyl pyrrolidone) doped with copper oxide nanoparticles", Int. J. Innov. Res. Sci. Eng. Technol., vol. 5, pp: 2033-2040 (2016).

[14] Z. Rzayev, B. Denizli and H. Denizli "Bioengineering functional Copolymers. XI. Copper (11)-Poly(N-vinyl-2-pyrrolidone-N-isopropylacrylamide) macro complexes"; J. Appl. Polym. Sci., vol.109, pp: 903-909 (2008).

[15] E. Abdelrazek, I. Elashmawi, A. Elkhodary and A. Yassin. Curr Appl Phys, vol.10, pp: 607 (2010).

[16] C. Hao, Y. Zhao, X. Dong, Y. Zhou, Y. Xu, D. Wang and G. Jiang; "Anomalous rheological behavior of poly(1-Vinyl-2-pyrrolidone) and CuCl 2 in solution and their interactions in solid composites"; J. Polym. Int., vol. 58, pp: 906 - 911 (2009).

[17] B. Rivas, E. Pereira and I. Viloslada; "Water-Soluble polymer-metal ion interactions" Prog. Polym. Sci., vol. 28, pp: 173-208 (2003). 
[18] E. Abdelrazeka, H. Ragabb and M. Abdelaziz; "Physical Characterization of Poly(vinyl pyrrolidone) and Gelatin Blend Films Doped with Magnesium Chloride Plastic and Polymer", Technology; vol. 2 Issue 1, (2013).

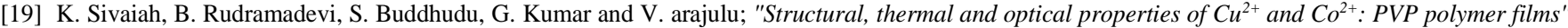
Indian J. Pure Appl. Phys., vol. 48, pp: 658 - 662 (2010).

[20] H. Fatima, T. Sandrine, M. Gilles and G. Jean-Luc, "Photooxidation of Poly(N-vinyl pyrrolidone), PVP in the Solid State and in Aqueous Solution", Polymer Deg-radation and Stability, vol. 94, No. 12, pp: 2257- 2266 (2009)

[21] Y. Kobayashi, A. Kosuge and M. Konno, "Fabrication of High Concentration Barium Titanate/Poly(vinyl pyrrolidone) Nano-Composite Thin Films and Their Dielectric Pro- perties", Applied Surface Science, vol. 255, No. 5, pp: 2723-2729 (2008).

[22] B. AL-Khayat and F. Awni, J. Am. Ceram. Soc. Bull. vol. 64, No. (4), pp: (598 - 601) (1985).

[23] R. Tintu, K. Saurav, K. Sulakshna, V. Nampoori, P. Krishnan and S. Thomas, "Ge $e_{28} S_{60} S b_{12} / P V A$ composite films for photoni application", Journal of Non-Oxide Glasses, vol. 2, No. (4), pp: 167-174 (2010).

[24] H. Haider, "Addition effect of poly (acryl amide) on the physical properties of Carboxymethyl Cellulose", M.Sc. Thesis, University of Babylon, (2014)

[25] A. Alkhayatt, A. Al-Azzawi, and Z. Alakayashi; "Rheological and optical characterization of Poly(vinyl pyrrolidone),PVP/Poly(ethylene glycol), PEG polymer blends"; Journal of Applied Physics, vol. 8, Issue 1, pp: 11-18 (2016).

[26] Teraoka, "Polymer Solutions", Polytechnic University, Brooklyn, John Wiley and Sons Inc., New York, p. 269 (2002).

[27] N. Saeed and A. Suhail, "Enhancement the Optical Properties of Zinc Sulfide Thin Films for Solar Cell Applications", Iraqi Journal of Sciences, vol. 53, No. (1); pp: 88-95 (2012).

[28] S. Nasraoui; "Investigation of the Effect of Temperature on the Optical and Electrical Properties of Hydroxyethyl Cellulose"; vol. 13, No. 3, (2002).

[29] A. Jangher; "Dielectric Properties of Poly (vinyl pyrrolidone) in 1,4-dioxane Solution"; M.Sc. Thesis, University of Tripoli, (2002).

[30] H. Raad, "Study the effects of the $\gamma$-rays on physical properties (Electrical, Optical, Rheological) of water soluble Poly(acryl amide)"; M.Sc. Thesis, University of Al-Mustansiriyah, (2006).

[31] R. Abbas; "Study of Gamma Radiation in Physical Properties of Polymer (PAAm)", M.Sc. Thesis, College of Science, Al- Mustansiryah University, (2006).

[32] J. Al-Bermany and S. Ahmad; "Study of Some Optical Properties of Carboxymethyl Cellulose Polymer by Adding Poly(vinyl alcohol)", J. of Babylon University, Pure and Applied Sciences, vol. 22, No.1, (2012). 